\title{
Evaluation of interaction dynamics of concurrent processes
}

\author{
Piotr Sobecki, ${ }^{*, * *}$ Jan T. Białasiewicz, ${ }^{* * *}, * * * *$ Nicholas Gross ${ }^{* * * *}$
}

\begin{abstract}
The purpose of this paper is to present the wavelet tools that enable the detection of temporal interactions of concurrent processes. In particular, the determination of interaction coherence of time-varying signals is achieved using a complex continuous wavelet transform. This paper has used electrocardiogram (ECG) and seismocardiogram (SCG) data set to show multiple continuous wavelet analysis techniques based on Morlet wavelet transform. MATLAB Graphical User Interface (GUI), developed in the reported research to assist in quick and simple data analysis, is presented. These software tools can discover the interaction dynamics of time-varying signals, hence they can reveal their correlation in phase and amplitude, as well as their non-linear interconnections. The user-friendly MATLAB GUI enables effective use of the developed software what enables to load two processes under investigation, make choice of the required processing parameters, and then perform the analysis. The software developed is a useful tool for researchers who have a need for investigation of interaction dynamics of concurrent processes.
\end{abstract}

K e y w or d s: Matlab graphical user Interface, signal processing, wavelet analysis

\section{Introduction}

In this paper, we present results of a project, in which we have developed wavelet-based analysis tools for obtaining meaningful information on interaction dynamics of concurrent processes. These tools can detect the shortlived temporal interactions. The investigated processes are represented by concurrent recordings obtained from a complex dynamic system, such as

- human body in which one would like to examine the relation between electrocardiogram (ECG) and seismocardiogram (SCG) measurements over the same period (that are the real data used for illustrative purposes in the present paper);

- wind turbine system, in which one is interested in the relationship between the turbulent wind pressure and the resulting bending moments developed in the blades of wind turbine rotor.

This research dates to the use of the Morlet Wavelet Transform (MWT) scalogram and coscalogram to examine the initial stiffness degradation of the turbine motor that was found to be primarily due to early presence of high frequency energy that causes excitation of higher structural modes leading to response coupling and energy exchange between modes, reported by Kelley et al [2,3]. Addison et al [1] used Morlet Wavelet Scalogram to detect a previously unknown coordinated contractility behavior of the atrium during ventricular fibrillation, a phenomenon which is not captured in a normal electrocardiogram. Similar applications of the MWT can also be found in Gonzlez et al [4] and Bialasiewicz et al [5]. Preliminary results of the research discussed in this paper are presented by Bialasiewicz [6]. Many researchers [7-14] introduced and investigated applications of additional mea- sures of interaction dynamics based on MWT scalogram and coscalogram. These measures include wavelet local correlation coefficient (WLCC), cross wavelet coherence function $(\mathrm{CWCF})$, wavelet coherence $(\mathrm{WC})$, and wavelet bicoherence (WB).
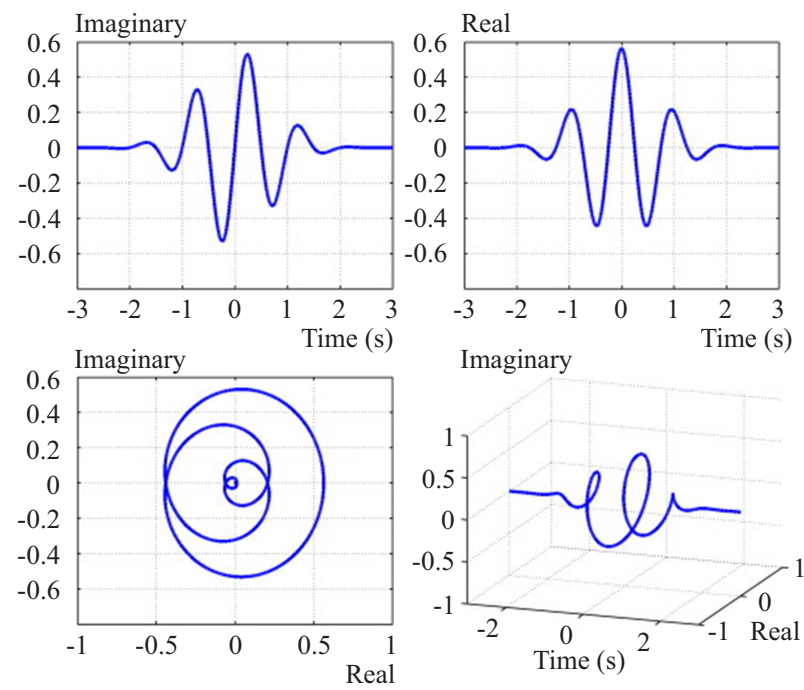

Fig. 1. Graphical representation of the complex-valued continuous Morlet wavelet

The Morlet wavelet, that is a Gaussian-windowed complex sinusoid, gives (due to the Gaussian's second order exponential decay) good time localization. The MWT is defined as

$$
W_{x}(s, \tau)=\frac{1}{\sqrt{s}} \int x(t) \psi^{*}\left(\frac{t-\tau}{s}\right) \mathrm{d} t
$$

where $\psi(t)=\pi^{-1 / 4} e^{j \omega_{0} t} e^{-1 / 2 t^{2}}$, and $x$ is the analyzed signal, $s$ is the scale.

* Faculty of Mathematics and Information Science, Warsaw University of Technology Koszykowa 75, 00-662 Warsaw, Poland, ** National Information Processing Institute, Warsaw, Poland aleja Niepodległości 188 b, 00-001 Warsaw, Poland, ptrsbck@gmail.com, *** Polish-Japanese Academy for Computer Technology, Warsaw, Poland, **** University of Colorado Denver, Denver, CO, USA 
The graphical representation of the Morlet wavelet is shown in Fig. 1. The complex-valued Morlet wavelet is used to obtain both amplitude and phase details. Consequently, MWT is able to investigate coherence/synchronization between processes recorded from a dynamic system, possibly at different/distant locations.

Using the data that represent investigated processes our software calculates MWT scalogram and coscalogram and generates graphical presentation of the results. These results enable the qualitative evaluation of interaction dynamics of investigated processes. In addition, we have implemented some algorithms that provide evaluations of mentioned above additional measures of interaction dynamics.

For effective use of our software, we have developed a user-friendly Graphical User Interface (GUI) that enables the user to load two processes under investigation, make choice of the required processing parameters and then perform the analysis. All obtained results are represented in a graphical window. In addition, we validated our software through processing the same data with our software and with the software developed by Grinsted et al [9]. As illustrated below, the obtained results are similar but in our plots the essential information is visible better. Examples of results presentations are given below for a real data set of biomedical signals. This choice of data reflects the authors' hope that the software developed will find numerous medical applications as a diagnostic instrument. Our software tools are readily available to the user through the internet link given below.

\section{Scalograms and coscalograms}

A local time-frequency energy density, which measures the energy of a signal $x$, in the so-called Heisenberg box of the scaled $\psi_{\tau, s}$ wavelet, is known as wavelet scalogram

$$
P_{W} x(\tau, s)=\left|W_{x}(s, \tau)\right|^{2}
$$

A local time-frequency energy density, which measures the cross-energy of two processes (that identifies their local correlation), known as wavelet coscalogram, is defined as

$$
P_{W} x y(s, \tau)=W_{x}(s, \tau) W_{y}^{*}(s, \tau) .
$$

Figure $2 \mathrm{a}$ shows the time series of the ECG and SCG signals. Figures 2b-d show Events 1 through 4 in the boxed areas. Notice Events 1 through 4 (numbered from left to right) shown when irregular behavior occurs on the ECG signal as reflected on its scalogram in Fig. 2b. Figure 2c shows the scalogram of the SCG signal. The color intensity represents the strength of a frequency within the SCG signal at a specific time. Events 1 through 4 show variation of sequences of slow increase and sudden decrease in hearth rate frequency. This is a display of respiratory sinus arrhythmia (RSA), a variation in hearth rate, occurring naturally during a breathing cycle. Figure 2d shows coscalogram of the ECG and SCG time series. Event 1 appears to not be as strongly correlated between the ECG and SCG as Events 3 and 4. This result is due to the SCG scalogram having less power during Event 1 compared to Events 3 and 4 . We used data from database of physiologic signals: http://www.physionet.org/physiobank/ $[15,7,8]$.

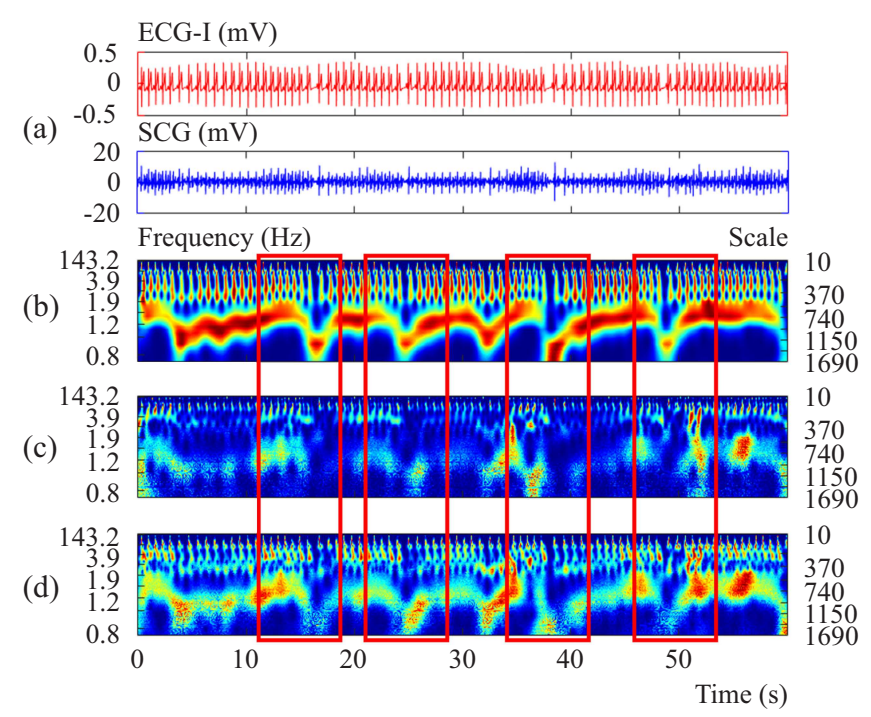

Fig. 2. (a) - ECG and SCG measured over 60 s period, scalograms of: (b) - ECG and, (c) - SCG data sets, (d) - coscalogram combining the ECG and SCG data; (b)-(d) show Events 1 through 4 in the boxed areas.

\section{Wavelet local correlation coefficient and cross wavelet coherence function}

Another technique to measure the correlation between two signals is using wavelet local correlation coefficient (WLCC) and cross wavelet coherence function (CWCF). WLCC and CWCF are

$$
\begin{gathered}
W L C C(s, \tau)=\frac{\operatorname{Re}\left[P_{W} x y(s, \tau)\right]}{\left|W_{x}(s, \tau)\right|\left|W_{y}(s, \tau)\right|}, \\
C W C F(s, \tau)=\frac{2\left|P_{W} x y(s, \tau)\right|^{2}}{\left|W_{x}(s, \tau)\right|^{4}+\left|W_{y}(s, \tau)\right|^{4}} .
\end{gathered}
$$

The former is a measure of phase correlation of two series in the time-scale (or time-frequency) domain, and the latter is a measure of the amplitude correlation in the time-scale (or time-frequency) domain.

The illustration of these two measures for our example is given in Fig. 3. Figure 3a shows the WLCC, which is the phase correlation. The color represents the amplitude strength of the coherence between the ECG and SCG signals. Notice the stripes in the lower frequency bands $(1.9<f<2.6 \mathrm{~Hz})$ and $(3.9<f<7.5 \mathrm{~Hz})$, which are within the same frequency region as that seen in the coscalogram of Fig. 2d. These stripes indicate that the signals have a phase correlation throughout the entire time series within these lower frequencies. Figure $3 \mathrm{~b}$ gives the $\mathrm{CWCF}$, where the color intensity represents the amplitude coherence. Again, notice that the most intense portions are within the lower frequency band, and that the 


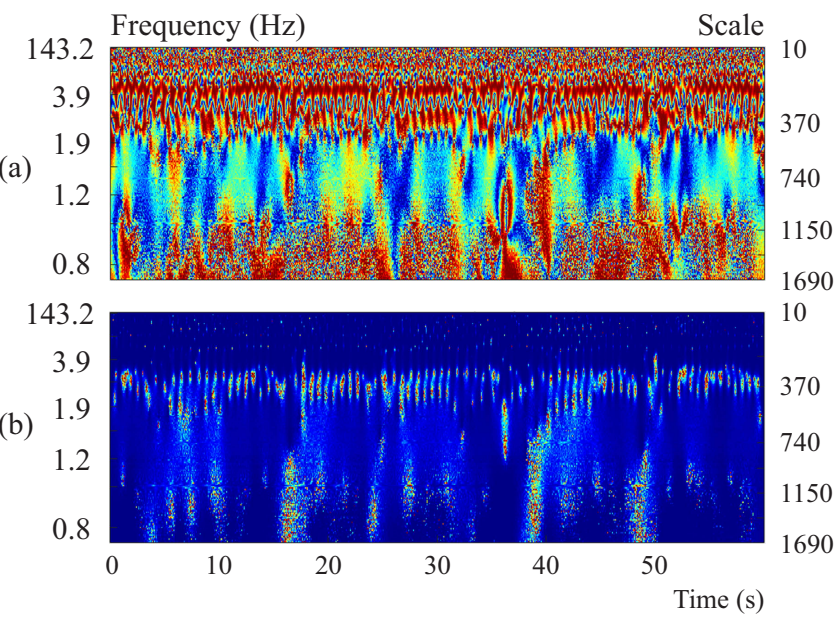

Fig. 3. The color represents the coherence of ECG and SCG signals: (a) - WLCC phase, (b) - CWCF amplitude strength.

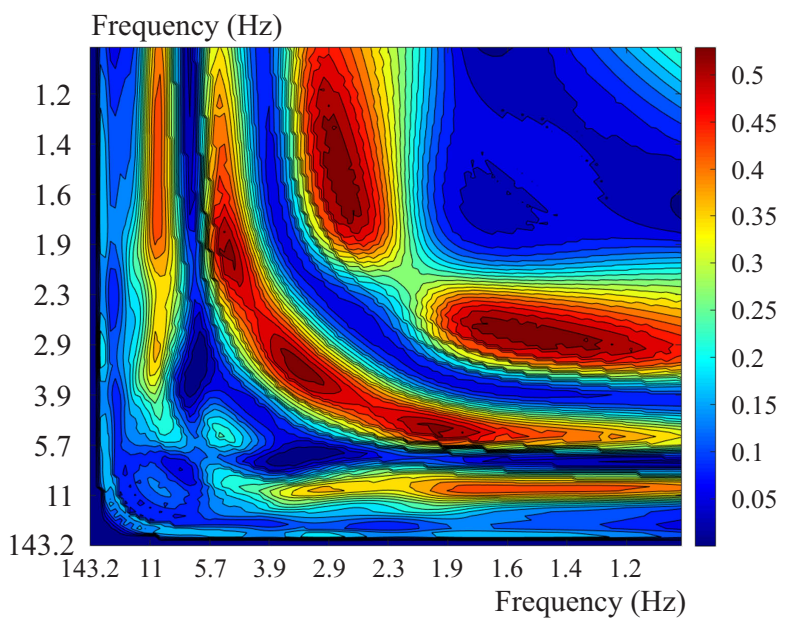

Fig. 4. WB during Event 3: the horizontal and vertical axes represent the ECG signal and the SCG signal frequencies

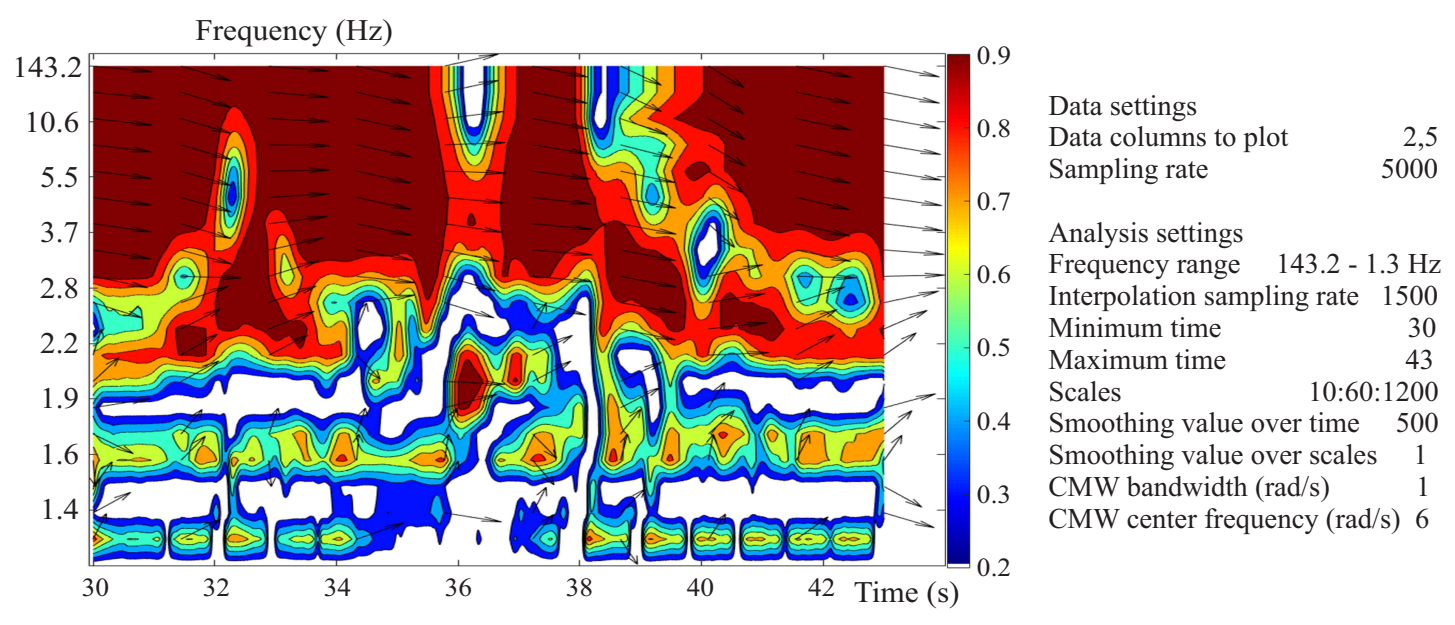

Fig. 5. Screenshot of the GUI running while plotting the coherence of ECG and SCG signals during Event 3

intense regions match the respective coscalogram portions within Events 1 through 4, as represented in Fig. 2d.

\section{Wavelet coherence}

The WC gives a localized view of the coherence strength of two time series signals, while reducing the noise through a smoothing operation. The WC and phase are defined as

$$
\begin{gathered}
W C=\frac{\left|S_{x y}^{\omega}(s, \tau)\right|^{2}}{S_{x x}^{\omega}(s, \tau) S_{y y}^{\omega}(s, \tau)}, \\
\varphi(s, \tau)=\tan ^{-1} \frac{\operatorname{Re}\left[S_{x y}^{\omega}(s, \tau)\right]}{\operatorname{Im}\left[S_{x y}^{\omega}(s, \tau)\right]}
\end{gathered}
$$

where

$$
\begin{aligned}
& S_{x y}^{\omega}(s, \tau)=\frac{1}{s} \int_{T} W_{x}(s, \tau) W_{y}^{*}(s, \tau) \mathrm{d} \tau, \\
& S_{x x}^{\omega}(s, \tau)=\frac{1}{s} \int_{T}\left|W_{x}(s, \tau)\right|^{2} \mathrm{~d} \tau, \\
& S_{y y}^{\omega}(s, \tau)=\frac{1}{s} \int_{T}\left|W_{y}(s, \tau)\right|^{2} \mathrm{~d} \tau, \\
& T=[\tau-\Delta \tau, \tau+\Delta \tau] .
\end{aligned}
$$

and can be employed over the data set with a smoothing parameter of $\Delta \tau$ that reduces the variance of noise. The coherence strength of the two time series signals is shown as color, and the superimposed arrows are used to indicate the coherence phase. Arrows that are pointing to right represent in-phase, arrows pointing up represent a positive phase shift of $90^{\circ}$ and arrows pointing down represent a negative phase shift of $90^{\circ}$ (as shown in Fig. 5). However, it is observed that Event 3 shows strongest coherence visible in lower frequency band $1<f<1.4 \mathrm{~Hz}$ which is most likely due to increased intensity seen in the scalogram of the SCG after Event 2. It can also be noted that most of the arrows are pointing relatively to the right, indicating "in phase" or strong coherence of the ECG and SCG signals that agrees with our findings regarding the frequency band $1.9<f<2.6 \mathrm{~Hz}$ in Fig. 3b, where the long stripes indicated that the signals had strong phase coherence.

\section{Wavelet bicoherence}

Wavelet bicoherence (WB) allows the two time series signals to be analyzed for phase coupling and non-linear interactions. 

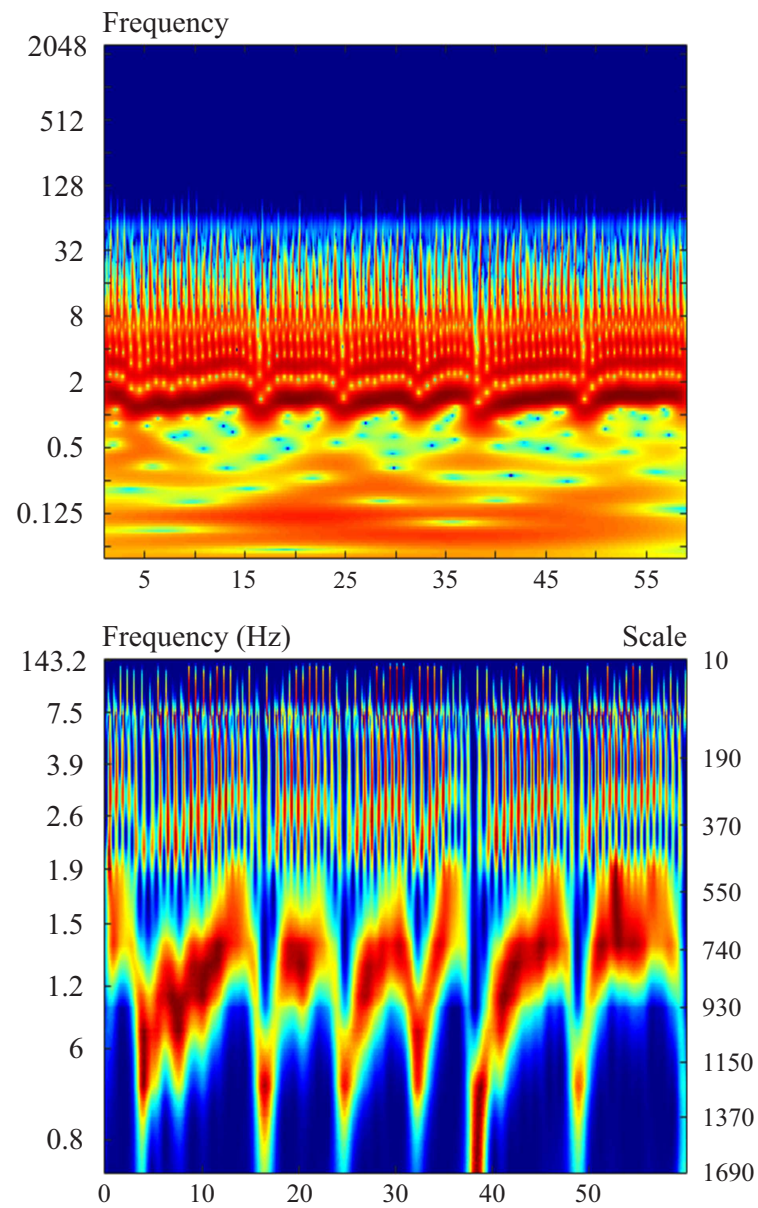

Fig. 6. ECG schalogram comparison (up Grinstead, et al )

The identity

$$
\cos (\alpha) \cos (\beta)=\frac{1}{2}[\cos (\alpha+\beta)+\cos (\alpha-\beta)]
$$

shows that two frequencies, $f_{1}$ and $f_{2}$, can create a new frequency $f=f_{1} \pm f_{2}$, through non-linear effects. This can then be transferred into scaling of the coefficients from the continuous wavelet transform, where $\frac{1}{s}=\frac{1}{s_{1}} \pm$ $\frac{1}{s_{2}}$.

The WB is defined as

$$
W B=\frac{\left|B_{x x y}^{W}\left(s_{1}, s_{2}\right)\right|}{\int_{T}\left|W_{x}\left(s_{1}, \tau\right) W_{x}\left(s_{2}, \tau\right)\right|^{2} \mathrm{~d} \tau \int_{T}\left|W_{y}(s, \tau)\right|^{2} \mathrm{~d} \tau}
$$

where

$$
B_{x x y}^{W}\left(s_{1}, s_{2}\right)=\int_{T} W_{x}\left(s_{1}, \tau\right) W_{x}\left(s_{2}, \tau\right) W_{y}^{*}(s, \tau) \mathrm{d} \tau
$$

and can be evaluated over any short or long time intervals. For the graphical illustration, we used our simultaneously recorded ECG and SCG signals. Figure 4 shows the wavelet bicoherence determined over a short time period of Event 3. Using bicoherence we are looking for phase coupling and non-linear interactions that take place between the investigasted signals during any event short time interval. In Fig. 4, the horizontal axes represent the ECG signal frequencies and the vertical axis represent the SCG signal frequency.

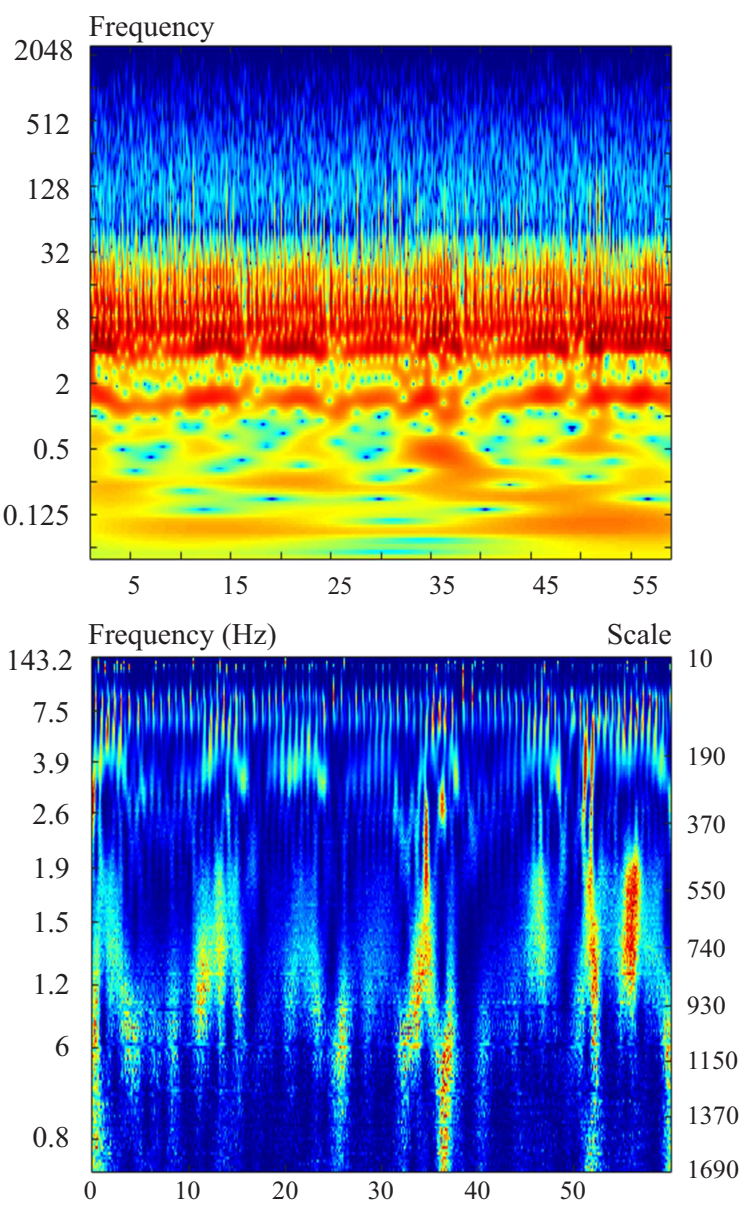

Fig. 7. SCG schalogram comparison (up Grinstead, et al)

\section{Wavelet analysis tools - GUI}

Running the program when analyzing twotime series signals and wanting to change only one parameter can become a monotonous task. To combat this tedious process, a MATLAB Graphical User Interface (GUI) was created. Fig ure 5 shows the window for the GUI, where the signals that are analyzed are identical as those in Fig. 2 except that the time interval of interest has been changed to $30<t<43$ seconds. Adjusting the time interval or any other parameter is very simple. The users can change any parameter they choose, then press the button for the plot they would like to have created. Sources of the developed program used in this article are available at https://github.com/piotrsobecki/TIDCPE.

\section{Discussion}

In this paper, we have used an ECG data set and a SCG data set to illustrate various continuous wavelet analysis techniques with the results that can be obtained using our programs that can be executed at the MATLAB's command line and/or using MATLAB's GUI that we have developed.

The same tools have been used by other researchers and similar results have been shown. Using some of the 
tools, discussed in this paper, we have processed the same data using our software and the software developed by Grinsted et al [9]. The comparison of results is illustrated in Fig. 6 and Fig. 7.

\section{Conclusion}

This paper has used an ECG data set and a SCG data set to show multiple continuous wavelet analysis techniques that can be used at MATLAB's command line or using MATLAB's GUI. When signals are dynamic over time, it is advantageous to use wavelet analysis instead of Fourier analysis. Wavelet analysis can maintain temporal characteristics whereas Fourier analysis removes the temporal characteristics.

It has been shown that these multiple techniques reveal interaction dynamics of time-varying signals (such as ECG and SCG time series signals) and how they can be correlated both in amplitude and phase along with phase coupling and non-linear interactions. Results for Events 1 through 4 were shown to have strong coherence between the ECG and SCG signals both in amplitude and phase. However, phase coupling and non-linear interactions were considered to be weak during these events. Finally, a MATLAB GUI was presented to assist in quick and simple data analysis.

\section{REFERENCES}

[1] P. S. Addison, J. N. Watson, G. R. Clegg, P. A. Steen and C. E. Robertson, "Finding Coordinated Atrial Activity During Ventricular Fibrillation Using Wavelet Decomposition", Analyzing Surface ECGs with A New Signal Analysis Technique to Better Understand Sudden Cardiac Death", IEEE Trans.Engineering Medicine and Biology, 2002.

$[2]$ N. D. Kelley, R. M. Osgood, J. T. Bialasiewicz and A. Jakubowski, "Using Wavelet Analysis to Assess Turbulence/ Rotor Interactions", Wind Energy, vol.3, no. 3, pp. 121-134, 2001.

[3] N. D. Kelley, B. J. Jonkman, J. T. Bialasiewicz, G. N. Scott and L. S. Redmond, "The Impact of Coherent Turbulence on Wind Turbine Aeroelastic Response and Its Simulation", Proc. AWEA Windpower '05, Denver, 2005.

[4] D. Gonzlez, J. T. Bialasiewicz, J. Balcells, and J. Gago, "WaveletBased Performance Evaluation of Power Converters Operating With Modulated Switching Frequency", IEEE Trans", Ind. Electron., vol. 55, no.8, pp. 3167-3176, August 2008.

[5] J. T. Bialasiewicz, D. Gonzlez, J. Gago, and J. Balcells, "WaveletBased Approach to Evaluation of Signal Integrity", IEEE Trans. Ind. Electron., vol. 60, no.10, pp. 4590-4598, October 2013.

[6] J. T. Bialasiewicz, "Application of Wavelet Scalogram and Coscalogram for Analysis of Biomedical Signals", Proceedings of the 2nd International Conference on Biomedical Engineering and Systems, Barcelona, Spain, July 2015, paper no. 333.

[7] M. A. García-González, A. Argelagós-Palau, M. Fernndez-Chimeno and J. Ramos-Castro, "A comparison of heartbeat detectors for the seismocardiogram", " Computing Cardiology Conference (CinC), 2013.

[8] M. A. García-González, A. Argelagós-Palau, M. Fernndez-Chimeno and J. Ramos-Castro, "Differences QRS Locations due to ECG Lead: Relationship with Breathing", XIII Mediterranean
Conference on Medical and Biological Engineering and Computing 2013, IFMBE Proceedings vol. 41, pp. 962-964, 2014.

[9] J. C. Grinsted, J. C. Moore and S. Jevreja, "Applications of the cross wavelet transform and wavelet coherence to geophysical time series", Nonlinear Processes Geophysics 2004, 11:561-566.

[10] Mohamed A. K. Elsayed, "Wavlet bicoherence analysis of wind-wave interaction", Ocean Engineering 33, 2006, pp. 458-470

[11] J. P. Lachaux, L. A. Rudrauf, A. Lutz, D. Cosmelli, M. L. V. Quyen and Martinerie J. Varela, "Estimating the time-course of coherence between single-trial brain signals: An introduction to wavelet coherence", Clin Neurophysiol 2002; 32:157-74.

12] H. R. M. Wolfgang, B. Hristoph, A. Matthias, W. Herbert and T. Edward, "Coherence of gamma-band EEG activity as a basis for associative learning", Nature 1999,397:43-46.

[13] E. Sanchez, T. Estrada, C. Hidalgo, B. Branas, B. Carreras and L. Garcia, "Wavelet bicoherence: a new turbulence analysis tool", Phys. Plasmas, 1995;2:301732.

[14] Xiaoli Li, Xin Yao, J. Fox and J. G. Je_erys, "Interaction dynamics of neuronal oscillations analysed using wavelet transforms", J. Neurosci. Methods, 2007, 160(1):178-185.

[15] A. L. Goldberger, L. A. N. Amaral, L. Glass, J. M. Hausdorff, P. Ch. Ivanov, R. G. Mark, J. E. Mietus, G. B. Moody, C.-K. Peng and H. E. Stanley, "PhysioBank, PhysioToolkit, and PhysioNet: Components of a New Research Resource for Complex Physiologic Signals", " Circulation 101, no.23, 2000.

Received 21 October 2016

Piotr Sobecki (MSc),received his BE and MSc degrees in Computer Science at the Polish Japanese Academy of Information Technology in Warsaw, currently a PhD candidate at Warsaw University of Technology, faculty of Mathematics and Information Sciences. He is currently working as scientific programmer in National Information Processing Institute. His research and publication interests include signal processing, machine learning and computer aided diagnosis systems.

Jan T. Białasiewicz received the MS degree from Warsaw University of Technology, Warsaw, Poland, and the PhD and DSc degrees from Silesian University of Technology, Gliwice, Poland, all in electrical engineering. Since 1985 he has been with the Electrical Engineering Department, University of Colorado Denver. He also is a Professor with the Polish-Japanese Institute of Information Technology, Warsaw, Poland. His research interests include control theory, modeling and identification of dynamic systems, renewable energy systems, and theory and applications of wavelets. He is the author of two books and numerous research publications. Dr. Bialasiewicz is a former Associate Editor of the IEEE Transactions on Industrial Electronics, a member of the IEEE Industrial Electronics Society Technical Committee on Renewable Energy Systems and a Registered Professional Engineer in the State of Colorado.

Nicholas Gross received his BS in electrical engineering from the University of Colorado Denver and is currently working towards his $\mathrm{PhD}$ in electrical and computer engineering at the Georgia Institute of Technology. His research interests include very low frequency radio-wave propagation, global characterization of the D-region ionosphere, and whistler waveparticle interactions in the magnetosphere. He also participates in the Georgia Tech Opportunity Research Scholars program, where he mentors a group of undergraduate research students working on an autonomous very low frequency receiver capable of measuring magnetic fields in the nanotesla range. 